\title{
Biophysica-A New International Open Access Journal to Integrate Across the Modern Biophysical Sciences and Engineering
}

\author{
Matthias Buck ${ }^{1, *(D)}$ and Victor Muñoz ${ }^{2, *}$ \\ 1 Department of Physiology and Biophysics, School of Medicine, Case Western Reserve University, \\ 10900 Euclid Avenue, Cleveland, OH 44106, USA \\ 2 Department of Bioengineering, Director NSF-CREST Center for Cellular and Biomolecular Machines (CCBM), \\ University of California Merced, 5200 North Lake Road, Merced, CA 95340, USA \\ * Correspondence: mxb150@case.edu (M.B.); vmunoz3@ucmerced.edu (V.M.)
}

check for updates

Citation: Buck, M.; Muñoz, V. Biophysica-A New International Open Access Journal to Integrate Across the Modern Biophysical Sciences and Engineering. Biophysica 2021, 1, 73-75. https://doi.org/ 10.3390/biophysica1010006

Received: 15 March 2021

Accepted: 16 March 2021

Published: 22 March 2021

Publisher's Note: MDPI stays neutral with regard to jurisdictional claims in published maps and institutional affiliations.

Copyright: (C) 2021 by the authors. Licensee MDPI, Basel, Switzerland. This article is an open access article distributed under the terms and conditions of the Creative Commons Attribution (CC BY) license (https:/ / creativecommons.org/licenses/by/ $4.0 /)$.
"What do Biophysicists talk about?"- "well, that depends who they are talking to. When they talk to Physicists, they talk about Biology. When they talk to Biologists, they talk about Physics ... and when they talk to fellow Biophysicists, among friends, they talk about life-discoveries to understand" (We will return to the wider meaning of this quib, at the very end).

Biophysics aims to understand in quantitative terms how biological systems function using methods and techniques borrowed from the physical and chemical sciences and applied math. Now that we are well into the 21st century, its original focus on certain molecular biological and physiology questions has widened to cover increasingly complex biological systems and processes. These biological systems are being studied across all scales (from molecules and cells, to tissues and living and interacting organisms) using a powerful palette of newly minted techniques and approaches. Examples of such enabling technologies go from new tools for the atomic-resolution structural analysis of larger biomolecular assemblies in their functioning states, the capability to investigate and manipulate biological systems at the single-particle or single-molecule level, the production of massive biological datasets through the deployment of "omics" approaches, the implementation of new advanced in vivo imaging and sensing methodologies, the accessibility to large-scale computer modeling capabilities, and the development of myriads of engineering applications in the areas of biotechnology, nanotechnology and biomaterials.

An inevitable byproduct of the expansion and success of modern biophysics has been the sprouting of many sub-fields, including molecular biophysics, cell biophysics, electrophysiology and structural biology, but also its intersection with other more broadly related areas such as bioinformatics, systems biology, synthetic biology, biomechanics, biological and biomedical imaging, physical neuroscience, biomolecular engineering, cellular and metabolic engineering and tissue engineering. Many new journals specialized in particular areas have also emerged alongside the expansion of the discipline, providing exciting opportunities for the targeted publication of new results. But a journal which brings a broader perspective to Biophysics and allows the intermingling of disciplines seemed to be missing.

In the midst of all that growth, we launch this new journal Biophysica, which aims to provide a multidisciplinary, open access forum to communicate, discuss and integrate all the aspects of modern biophysical sciences and engineering in a broad sense. The long-term goal of the journal is to serve as a general conduit for the exchange of results across biophysics-related subdisciplines. Biophysica will publish the results of original research, reviews, short communications, and commentaries. Of particular interest to the journal are: (1) results that involve the development of novel technologies and analytical procedures of potentially broad biophysical applicability; (2) the discovery of emerging 
phenomena/principles of general biophysical relevance; (3) contributions that shed new mechanistic/quantitative light into long-standing biological problems; and (4) contributions that integrate multiple approaches across the subdisciplines of modern biophysics. As an open access online journal, Biophysica does not have restrictions on paper length, and hence authors can present detailed methods and extensive experimental data (of course being cognizant of the attention span of reviewers and readers). The journal will also promote Special Issues aimed to provide more detailed information of specific biophysical subfields or important current research topics.

We will consider papers that use a biophysics approach to advance our understanding of a biologically significant problem or develop a new technology. Work that makes incremental contributions, or that is simply descriptive is not suitable for this journal. In terms of scope, Biophysics is interested in the following areas:

1. Structure and dynamics of biomolecules and their assemblies: proteins, nucleic acids, biological membranes and supramolecular assemblies;

2. Biomolecular Machines: enzymes, motors, channels, receptors, transporters, circadian clocks, and other biological nanomachines;

3. Genetics and Gene Expression mechanisms: genome architecture, chromatin structure and dynamics, gene expression, DNA repair, genome editing, epigenetics;

4. Cell Biophysics: electrophysiology, cell division, signaling and migration, cell differentiation, tissue organization and tissue engineering;

5. Biophysical Techniques and Instrumentation: methods for the high-resolution structural and dynamic analysis of biomolecular systems, single-molecule spectroscopy and manipulation, biological imaging methods and applications, biosensor engineering and design;

6. Theory and Modeling of Biological Systems: applications of statistical mechanical modeling, computer simulations, or methods for the quantitative analysis of large biological datasets aiming at the rationalization or prediction of experimentally measured phenomena.

Other areas of interest are:

7. Biomechanics, Sensing and Signaling;

8. Quantitative Synthetic Biology;

9. Bioengineering, Nanotechnology and Biomaterials.

Finally, as our quib at the start indicated, a journal and the process of evaluating biophysical research for publication is also a community. It involves the time and broad insight of academic and industry based associate editors and the dedication of countless front line peer reviewers. The process of putting papers into Biophysica thus brings us together in a common endeavor with valuable benefits:

Let the exchange of ideas, gathering of stimulating experience and discovery of new insights -useful for our own research- begin!

Conflicts of Interest: The author declares no conflict of interest. 


\section{Short Biography of Authors}

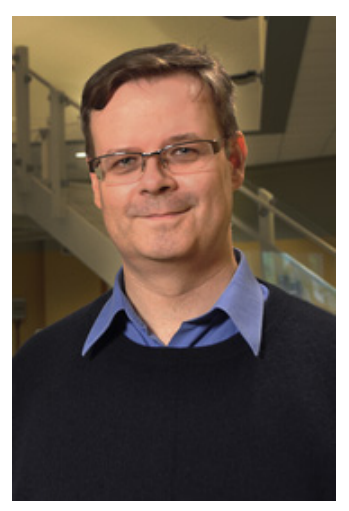

\section{Matthias Buck}

Prof. Matthias Buck, M.A., D.Phil. is Professor in the Departments of Physiology and Bio-physics, Neuroscience and Pharmacology at Case Western Reserve University School of Medicine. He is a native of Hamburg, and prior to starting his independent career in Cleveland in 2002, he did his undergraduate studies at the University of Cambridge, and obtained his doctorate from Oxford University, followed by postdoctoral work at Har-vard University and the Memorial Sloan Kettering Cancer Center. He is the Director of the North East Ohio High Field Nuclear Magnetic Resonance Facility. His research inter-est focuses on the characterization of protein-protein and protein-membrane interactions at the molecular level, using structural biology and biophysical tools as well as computa-tional modeling and simulations. His team is currently working on the regulatory mech-anisms of Plexin and Eph transmembrane receptors as well as on small membrane bound Ras and Rho GTPases. He enjoys traveling and when he is not working is playing with his two young daughters, likes EDM, Wine, Photography and Poetry (Google Scholar h-index = 33).

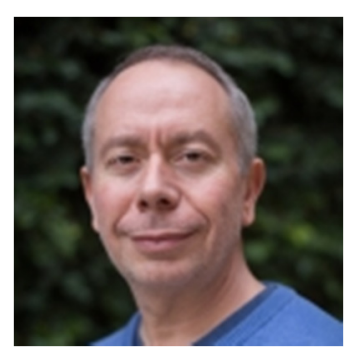

\section{Victor Muñoz}

Prof. Victor Muñoz is a Professor of Bioengineering at the University of California Merced and Director of the NSF-CREST Center for Cellular and Biomolecular Machines. He was born in Liège (Belgium) and raised in Madrid (Spain). He carried out his PhD at the European Molecular Biology Laboratory (EMBL) in Heidelberg, Germany, and his postdoctoral training at the US National Institutes of Health in Bethesda, Maryland. During his independent career he has been as at the University of Maryland as Assistant and Associate Professor (2000-2007), at the Spanish National Research Council as Re-search Professor (2007-2014), and at UC Merced since 2014. He is a Packard Fellow (2001), Searle Scholar (2002) and EMBO member (2009). His research area is experi-mental and computational protein biophysics and biomolecular engineering. Current re-search interests are in protein folding, binding and function, eukaryotic gene expression, calcium signaling and intrinsically disordered proteins, as well as cool technology-based projects in advanced protein biosensors, allosteric macromolecular assemblies, and mo-lecular diagnostics. In his leisure time he enjoys hiking with his two huskies, riding his Ducati and sky diving (Google Scholar h-index $=49$ ). 